\title{
Drosophila viewer: a program on the formal genetics, anatomy and developmental biology of Drosophila melanogaster for students and specialists
}

\author{
P.A. Otto
}

\section{INTRODUCTION}

The present Windows-based program was compiled in Visual Basic (Microsoft, Inc.) and presents data and pictures on the formal genetics, anatomy, and developmental biology of Drosophila melanogaster. With more than 150 plain and formatted texts, adapted from "Genetic Variations of Drosophila melanogaster" by D.L. Lindsley and E.H. Grell (1972), the program provides details of phenotypes of common genic mutants of the fly, as well as most India ink illustrations made by Mrs. Edith M. Wallace, Professor Thomas Hunt Morgan's illustrator. Illustrations of the external morphology of Drosophila were adapted from Ferris' chapter in the book "Biology of Drosophila" edited by M. Demerec (1950). The program provides color photomicrographs of specimens that have been cleared, whole-mounted, photographed and digitalized, and standardized color illustrations of the mutant phenotypes and of anatomical details. The program will be updated with new material (illustrations, photos, and text) as they become available. Future updates can be carried out by the addition of accessory files.

The program was designed to run in any IBM-PC-compatible computer with a $386-\mathrm{MHz}$ (or higher) processor and Windows 3.1 or later versions, a 16-color VGA monitor (at least 256 colors are necessary, however, to visualize the photographs), and $12 \mathrm{MB}$ of hard disk space to accommodate the accessory files. Since the program was created for a screen resolution of $640 \times 480$, users of recent versions of Windows (95 or higher) should disable the inferior taskbar of the computer graphic interface before running the program. The present version of the program in the compressed self installation form (with an approximate size of $3 \mathrm{MB}$, including all the accessory files and the library file VBRUN300.dll, necessary to run programs compiled in Visual Basic V.3.0) can be obtained free of charge directly from the author via his internet site (http:/ /www.ib.usp.br/ otto/software.htm) or requested by E-mail (otto@usp.br). Although freely distributed, the content of the program is the intellectual property of the author; as such, any use made of the material included in it should contain an explicit reference to its origin [Otto PA, Drosophila Viewer Program (V. 6.0), Department of Biology, University of São Paulo, 2000]. Feedbacks from the Drosophila community and from other geneticists are invited and will be used to correct any flaws and to improve the program.

\section{DESCRIPTION OF THE PROGRAM}

The illustrations shown in the figures are screen images obtained from the program and describe its functioning (Figures 1-12).

\section{ACKNOWLEDGMENTS}

The following persons and institutions are acknowledged for their direct or indirect help in developing the program: Paulo A.S. Nuin for many helpful suggestions related to the program code; Mrs. Elizabeth Powers, Cold Spring Harbor Laboratory Press, for permitting the use and adaptation of figures from Demerec $\mathrm{M}$. (Ed.), The Biology of Drosophila. Wiley \& Sons, New York, 1950 (reprinted by Cold Spring Harbor Laboratory Press, Plainview, 1994); Dr. Daniel L. Lindsley for permitting the reproduction of the public domain pictures and text from Lindsley D.M. and Grell E.H., Genetic Variations of Drosophila melanogaster, Carnegie Institution of Washington Publication No. 627, Washington, 1972; Drs. Carlos R. Vilela and Louis B. Klaczko (as well as other Drosophilist colleagues) for providing specimens of $D$. melanogaster and for their comments and suggestions; Dr. Andre L.P. Perondini, for his patience in teaching me several basic Drosophila handling techniques and for the use of his laboratory facilities. Publication supported by FAPESP.

\section{REFERENCES}

Demerec, M. (Ed.). (1950). The Biology of Drosophila. Wiley \& Sons, New York (reprinted by Cold Spring Harbor Laboratory Press, Plainview, 1994), pp. 632.

Lindsley, D.M. and Grell, E.H. (1972). Genetic Variations of Drosophila melanogaster. Carnegie Institution of Washington Publication No. 627, Washington, pp. 472.

(Received July 13, 2000) 


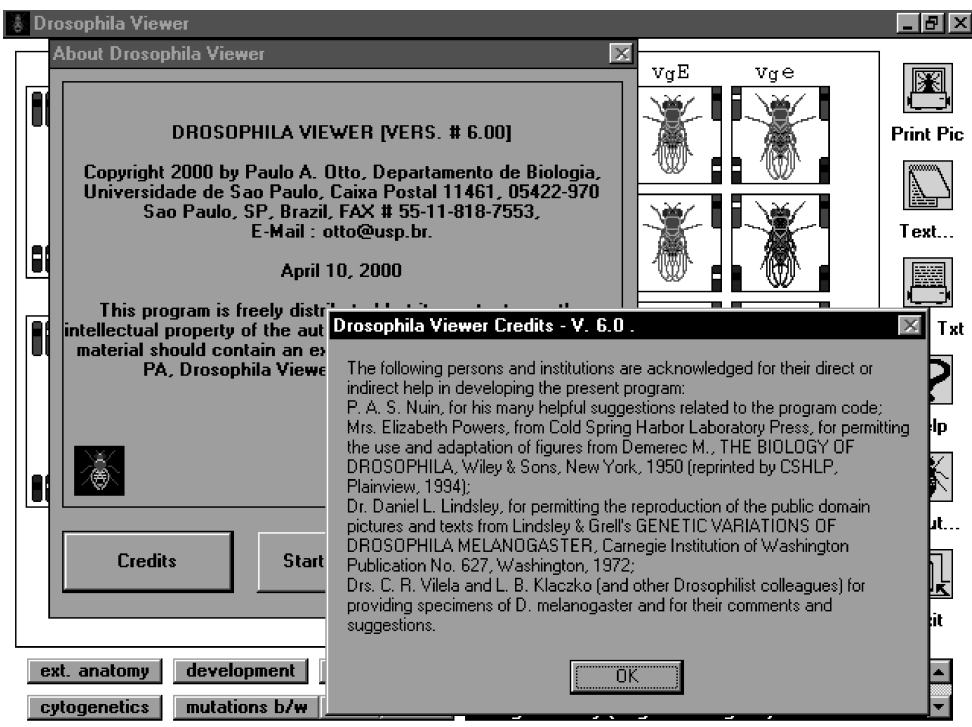

If Drosophila Viewer

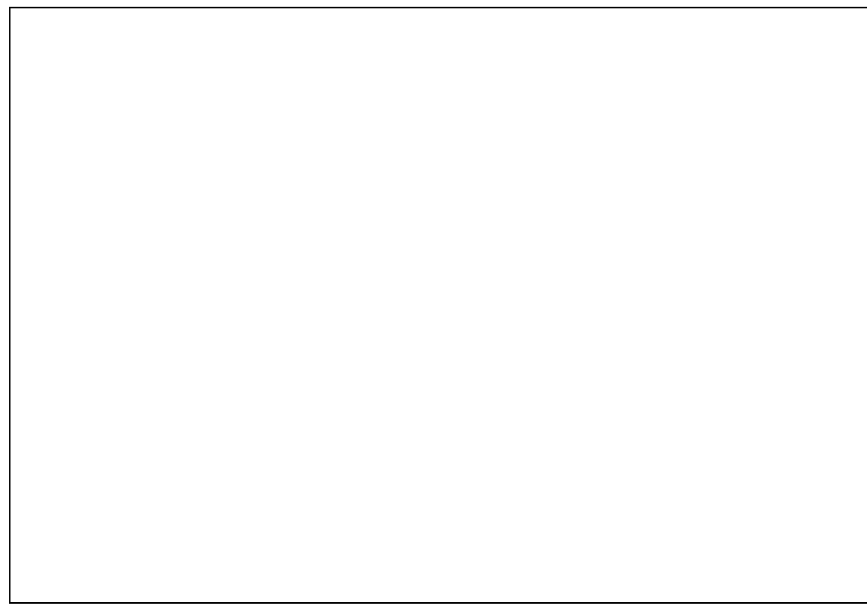

development crossings

\begin{tabular}{|l|l|l|l|}
\hline cylogenetics & mutations $\mathrm{b} / \mathrm{w}$ & col & pho \\
\hline
\end{tabular}

\section{F回区}

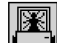

Print Pic

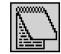

Text...

豎

Print Ixt

?

Help

粪

About.

$-]_{\mathrm{K}}$

Exit
If Drosophila Viewer

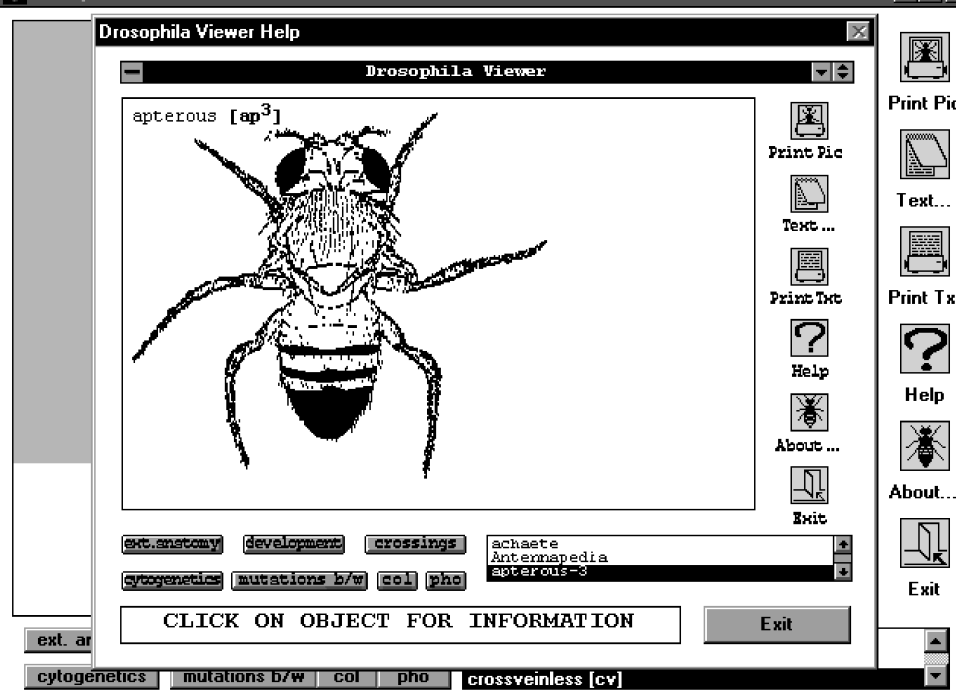

Figure 1 - Opening page of the program, with credits.

Figure 2 - Main graphic interface of the program, before the options given by the command buttons at the bottom and pictures on the right are chosen.

$\overline{\text { Figure } 3 \text { - Help interface of the program giving summarized }}$ instructions to run it and explanations about its functioning. 


\section{Drosophila Viewer}

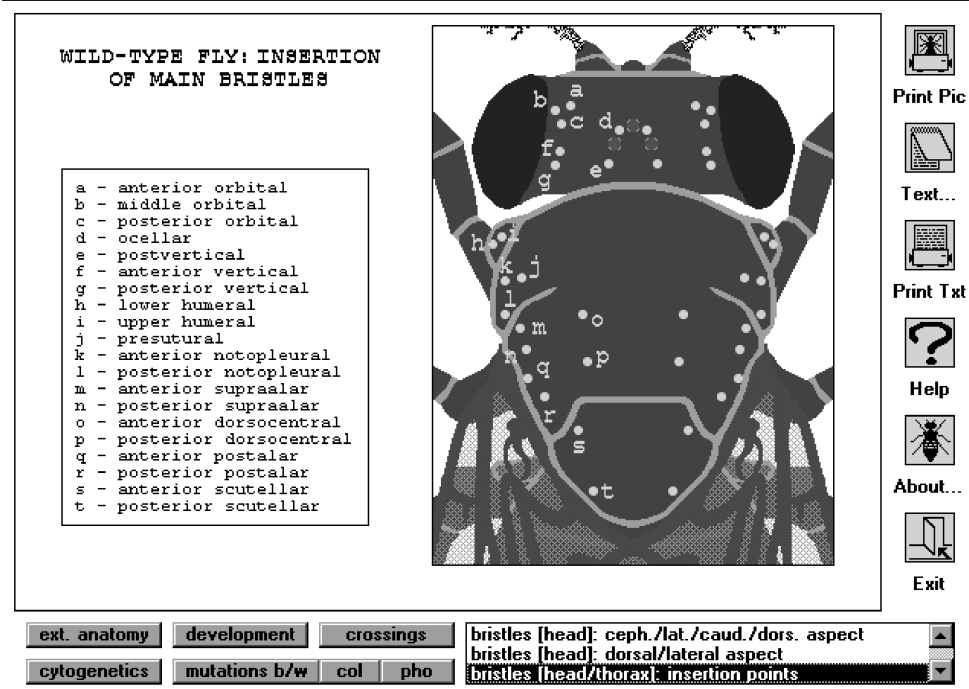

Drosophila Viewer

- 回区
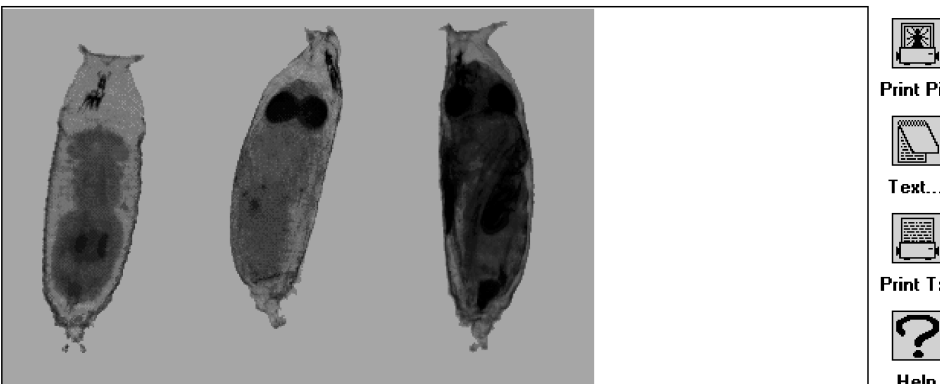

Print Pic

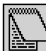

Text.

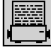

Print Txt
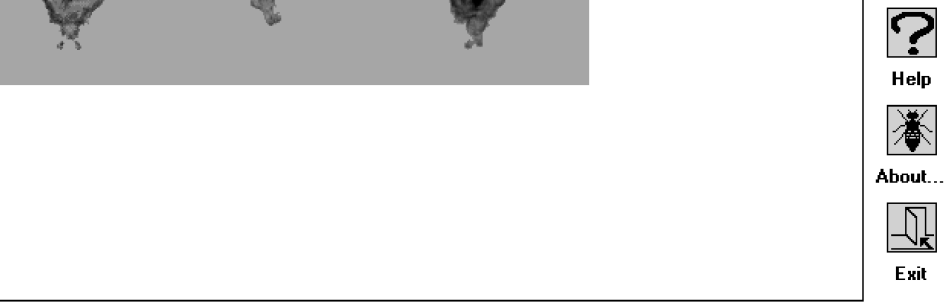

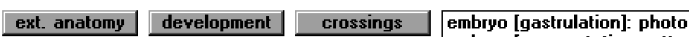
\begin{tabular}{|l|l|l|l|l|}
\hline cytogenetics & mutations b/w & col & pho \\
\hline
\end{tabular}
Figure 4 - Color drawing (original) showing the insertion of main bristles of head and thorax in a wild-type fly. The picture was selected by clicking the item in the list shown on the right after pressing the command button "ext(ernal). anatomy."

Figure 5 - Color photomicrograph showing three different stages of pupal development in Drosophila (original preparation and photograph). The picture was selected by clicking the item in the list shown on the right after pressing the command button "development."

- 回区

\section{Drosophila Viewer}
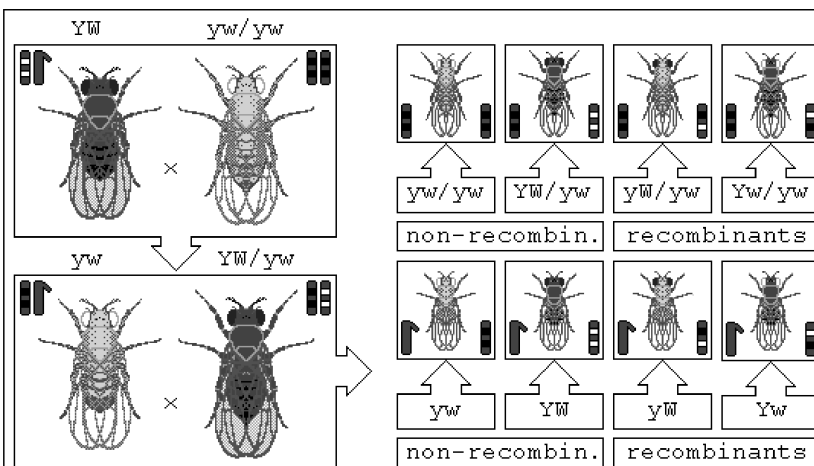

non-recombin. recombinants

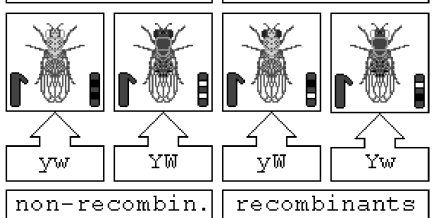

non-recombin. recombinants
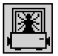

Print Pic

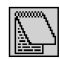

Text.

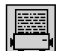

Print Txt

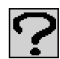

Help

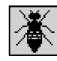

About...

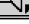

Exit

ext. anatomy development crossings Bar [m B x f Bb]

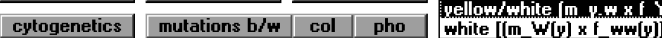

Figure 6 - Diagram illustrating non-independent assortment of two X-linked mutant recessive characters, yellow and white. Original drawing, based on Morgan et al. (The Mechanism of Mendelian Heredity, Holt, N. York, 1923). The picture was selected by clicking the item in the option list shown on the right after pressing the command button "crossings." 


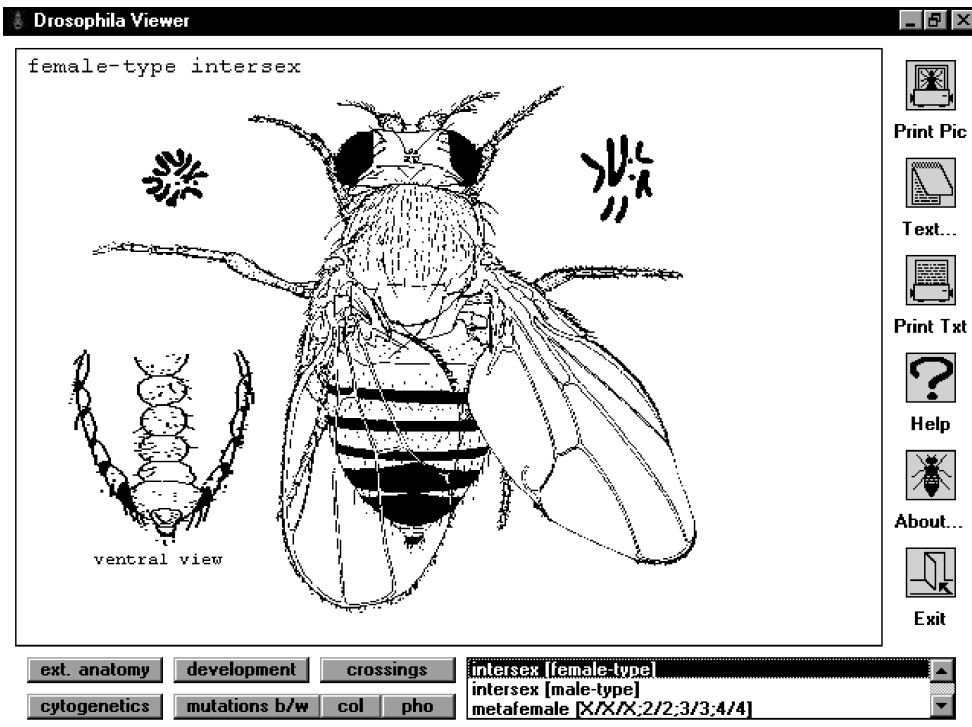

Drosophila Viewer

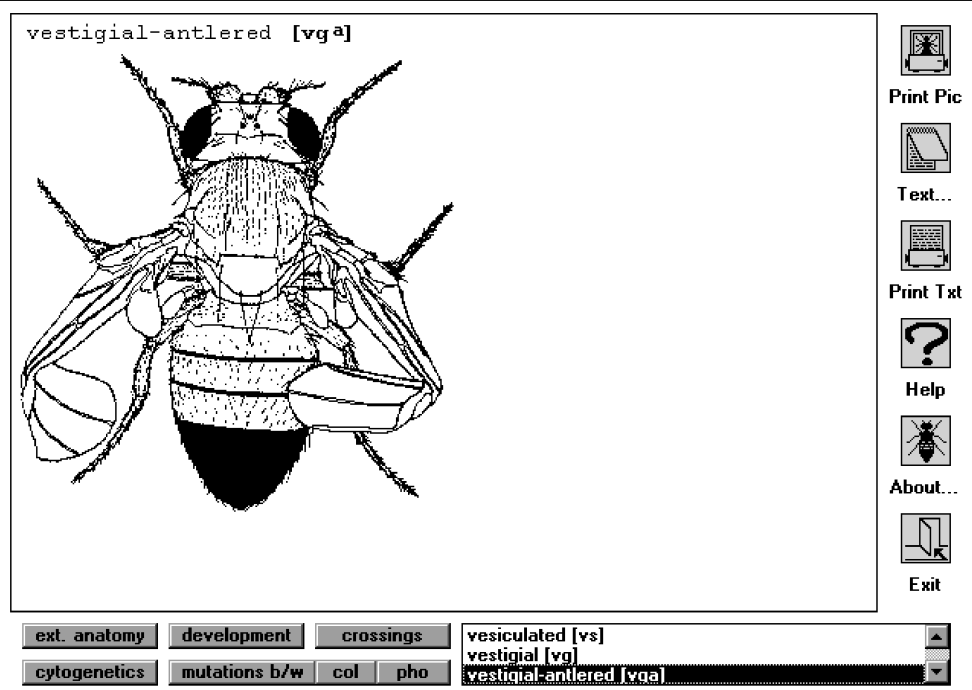

Drosophila Viewer

D回区

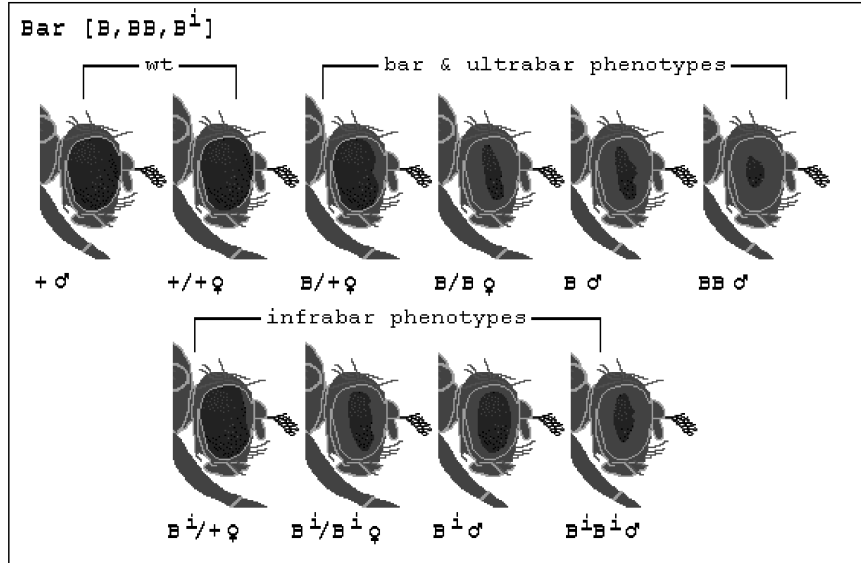

蒾圈

Print Pic

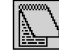

Text.

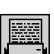

Print Txt

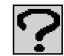

Help

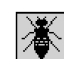

About.

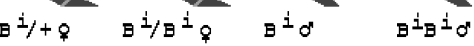

Exit

Figure 7 - Female-type intersex with a ventral view detail shown on the left. Original drawing by Edith M. Wallace. The picture was selected by clicking the item in the option list shown on the right after pressing the command button "cytogenetics."

Figure 8 - Example of an India ink black and white illustration showing the autosomal recessive vestigial-antlered phenotype. Original drawing by Edith $\mathrm{M}$. Wallace. The picture was selected by clicking the item in the option list shown on the right after pressing the command button "mutations b(lack)/w(hite)."

Figure 9 - Diagram illustrating the various X-linked bar phenotypes (original). The picture was selected by clicking the item in the option list shown on the right after pressing the command button "(mutations) col(or)." 


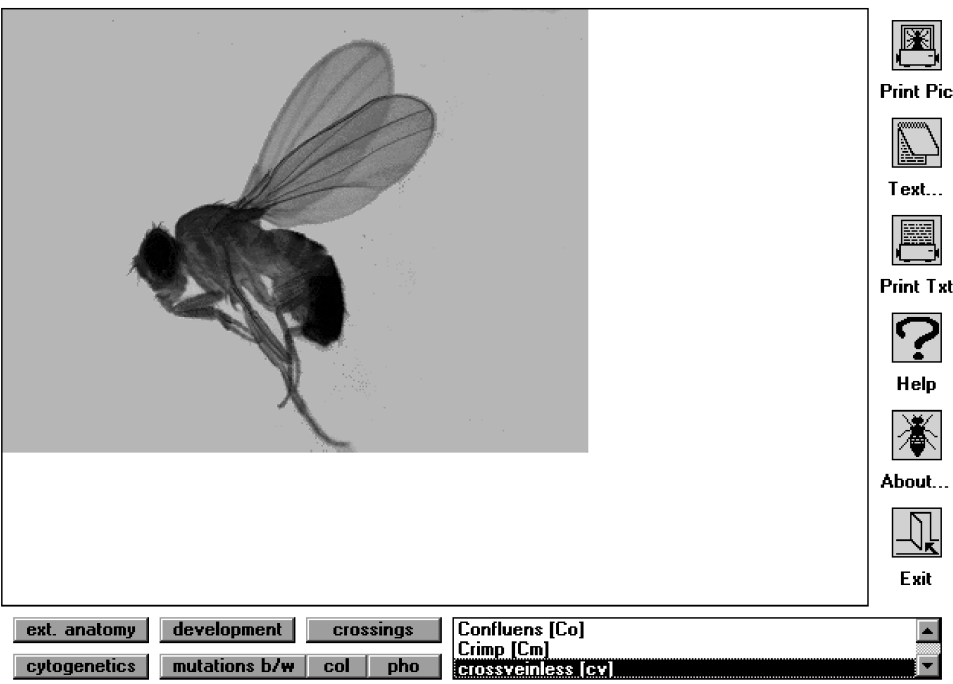

\section{BLACK $[\mathrm{b}]$}

Lindsley \& Grell, $197 z$, p. $z z$

location: $2-48.5$.

origin: Spontaneous.

Print Pic

discoverer: Morgan, $10 \mathrm{j}$. Morgan, 1919, Carnegie Inst. Wash. Publ. No. 278: 144

referen.

phenotype: Black pigment on body and tarsi and along wing veins, darkening

with age. Heterozygote shows somewhat darker trident, but is never confused

with homozygote. Puparium usually somewhat lighter than wild type and newly

emerged flies not clearly distinguishable from wild type (Waddington, 1941

proc. Zool. Soc. London, Ser. A 111: 173-80\%. Tyrosinase formed in adult

(Horowitz). RKI in aged flies.

cytology: Salivary chromosomes apparently normal. placed in region between

$34 \mathrm{E} 5$ and $35 \mathrm{D} 1$, on basis of its inclusion in $\mathrm{D} f(2 \mathrm{~L}) 64 \mathrm{j}=\mathrm{D} f(2 \mathrm{~L}) 34 \mathrm{E} 5-\mathrm{F} 1 ; 35 \mathrm{C} 3-\mathrm{D} 1$

(B. H. Grell).

color figure: P. A. Otto (original

photograph: P. A. Otto (original

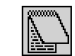

Exit Txt

BLACK $[\mathrm{b}]$

indsley s Grell, $1972,0.22$

location: $2-48.5$.

origin: Spontaneous.

discoverer: Morgan, $10 \mathrm{j}$.

references: Bridges and Morgan, 1919, Carnegie Inst. Wash. Publ. No. 278: 144

(fig.) :

phenotype: Black piquent on bodv and tarsi and along wing veins, darkening

with age. Hetero Instructions for printing DROSOPHILA VIEWER text files

with homozygote.

emerged flies no

(Horowitz) PR1

cytology: Saliva

$34 \mathrm{E5}$ and $35 \mathrm{DL}$,

(B. H. Grelli).

color ficure: $p$

photograph: p.
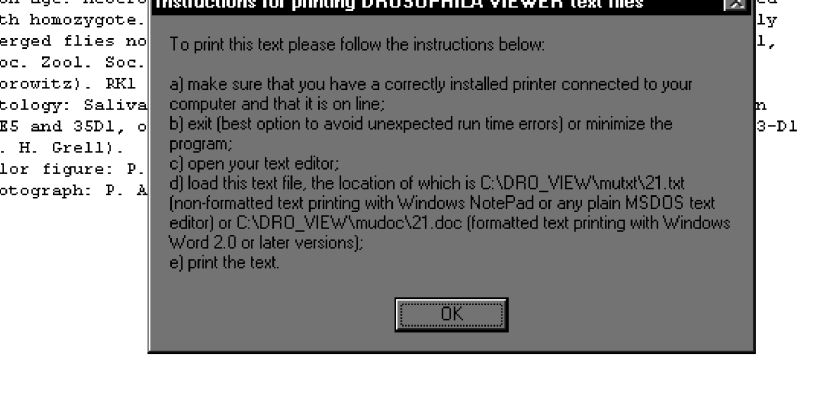

Figure 10 - Color photomicrograph of whole-mounted preparation of a fly presenting the $\mathrm{X}$-linked recessive mutation crossveinless (original photo and preparation). The picture was selected by clicking the item in the option list shown on the right after pressing the command button "(mutations) pho(to)."

Figure 11 - Sample text obtained by clicking the figure "Text..." [when clicked, the figure's label toggles to "Exit $t(e) x t$ "]. The text corresponds to the item selected from the mutations option list shown at the bottom left.

Figure 12 - Instructions for printing the selected text. When a text is open in the main window, the keying of the figure "Print $\mathrm{T}(\mathrm{e}) \mathrm{xt}$ " shows a window with summary procedures to obtain plain or formatted text. Alternatively, the text shown on the screen can be highlighted with the left mouse button and copied $(\mathrm{ctrl}+\mathrm{C})$ to the computer clipboard to be transferred to a word processor and printed later. 
